\title{
MHD Natural Convection with Convective Surface Boundary Condition over a Flat Plate
}

\author{
Mohammad M. Rashidi, ${ }^{1,2}$ Mohammad Ferdows, ${ }^{3}$ \\ Amir Basiri Parsa, ${ }^{4}$ and Shirley Abelman ${ }^{5}$ \\ ${ }^{1}$ University of Michigan-Shanghai Jiao Tong University Joint Institute, Shanghai Jiao Tong University, Shanghai 200000, China \\ ${ }^{2}$ Mechanical Engineering Department, Engineering Faculty of Bu-Ali Sina University, Hamedan 65141, Iran \\ ${ }^{3}$ Department of Mathematics, Dhaka University, Dhaka 1000, Bangladesh \\ ${ }^{4}$ Young Researchers and Elites Club, Islamic Azad University, Hamadan Branch, Hamadan 65141, Iran \\ ${ }^{5}$ Centre for Differential Equations, Continuum Mechanics and Applications, School of Computational and Applied Mathematics, \\ University of the Witwatersrand, Johannesburg, Private Bag 3, Wits 2050, South Africa
}

Correspondence should be addressed to Shirley Abelman; shirley.abelman@wits.ac.za

Received 15 March 2014; Revised 18 May 2014; Accepted 20 May 2014; Published 16 June 2014

Academic Editor: Rehana Naz

Copyright (c) 2014 Mohammad M. Rashidi et al. This is an open access article distributed under the Creative Commons Attribution License, which permits unrestricted use, distribution, and reproduction in any medium, provided the original work is properly cited.

\begin{abstract}
We apply the one parameter continuous group method to investigate similarity solutions of magnetohydrodynamic (MHD) heat and mass transfer flow of a steady viscous incompressible fluid over a flat plate. By using the one parameter group method, similarity transformations and corresponding similarity representations are presented. A convective boundary condition is applied instead of the usual boundary conditions of constant surface temperature or constant heat flux. In addition it is assumed that viscosity, thermal conductivity, and concentration diffusivity vary linearly. Our study indicates that a similarity solution is possible if the convective heat transfer related to the hot fluid on the lower surface of the plate is directly proportional to $(\bar{x})^{-1 / 2}$ where $\bar{x}$ is the distance from the leading edge of the solid surface. Numerical solutions of the ordinary differential equations are obtained by the Keller Box method for different values of the controlling parameters associated with the problem.
\end{abstract}

\section{Introduction}

A review of the literature shows that to the best of our knowledge not much research has been reported on MHD flow over a flat plate with convective surface boundary conditions by applying the one parameter continuous group method. For this problem we apply similarity transformations on the partial differential equations. The transformed nonlinear coupled ordinary differential equations are solved numerically by the Keller Box method for different values of controlling parameters.

Analysis of natural phenomena usually leads to partial differential equations and nonlinear ordinary differential equations. Nonlinear differential equations appear in physics, applied mathematics, and engineering sciences. In most cases for these problems exact solutions cannot be obtained. One of the most widely used applications of nonlinear differential equations is boundary-layer problems. Fluid flow and heat transfer are a relevant problem in many industrial processes such as metal and polymer extrusion processes, glass-fiber and paper production, manufacture and drawing of plastics and rubber sheets, and crystal growing. Magnetohydrodynamics (MHD) is the flow of an electrically conducting fluid in the presence of a magnetic field. This effect is of importance in various areas of technology and engineering such as MHD flow meters, MHD power generation, and MHD pumps [1-4]. The study of the interaction of conducting fluids with electromagnetic phenomena is important and such problems have received much attention from many researchers. Mukhopadhyay et al. [5], Andersson [6], Rashidi et al. [7], and Parsa et al. [8] investigated the effect of magnetic field over a stretching surface in various states. Numerical results for MHD free convection flow over a wedge in the presence of a magnetic field were presented by Watanabe and Pop [9]. Kumari and Nath [10] studied unsteady MHD viscous flow and heat transfer of Newtonian fluids induced by 
an impulsively stretched plane surface in two lateral directions by employing the homotopy analysis method. Rashidi et al. [1] solved the governing equations of suction and injection effects on the free convection boundary-layer flow over a vertical cylinder. In addition, a complete investigation of MHD studies and their technological applications was undertaken by Moreau [11]. Several interesting computational studies of reactive MHD boundary-layer flows with heat and mass transfer have appeared in recent years [12-15]. Effects of anisotropic scattering on steady nonsimilar free convective radiative hydromagnetic boundary-layer flow over a diffuse reflecting surface and solution of a separate equation for the magnetic field distribution were presented by Chen [16]. Ishak [17] studied steady laminar boundary-layer flow and heat transfer over a stationary permeable flat plate immersed in a uniform free stream with convective boundary condition. The problem of a vertical plate with convective boundary conditions was considered by Makinde [18]. Rashidi et al. [19] presented the first and second law analyses of an electrically conducting fluid past a rotating disk in the presence of a uniform vertical magnetic field by using the homotopy analysis method (HAM) and then applied artificial neural networks (ANN) and the particle swarm optimization (PSO) algorithm to minimize the entropy generation.

The main objective in this paper is to investigate similarity solutions and scaling transformations of MHD heat and mass transfer flow of a steady viscous incompressible fluid over a flat plate with convective surface boundary conditions by using the one parameter continuous group method. A convective boundary condition instead of the commonly used constant surface temperature or constant heat flux boundary conditions is applied. The governing boundarylayer equations are transformed to a two-point boundary value problem in similarity variables, and the problem is solved numerically by the Keller Box method. The effects of governing parameters on fluid velocity, temperature, and particle concentration are investigated and shown graphically.

\section{Mathematical Formulation of the Problem}

The problem of two-dimensional steady MHD heat and mass transfer laminar flow of a viscous incompressible and electrically conducting fluid past a flat plate is considered. The $\bar{x}$ axis is taken along the plate and the $\bar{y}$ axis is normal to the plate. The gravitation acceleration vector is parallel to plate. A magnetic field of uniform strength $B_{0}$ is applied perpendicular to the direction of the plate. The viscosity, thermal conductivity, and concentration diffusivity of fluid are assumed to vary linearly. The top surface of the plate is kept at uniform temperature $T_{w}$ which is assumed to be greater than the full stream temperature $T_{\infty}$. The species concentration $C_{w}$ at the surface is uniform and the full stream concentration is $C_{\infty}$. The bottom surface of the plate is heated by convection from a hot fluid of temperature $T_{f}$ which provides a heat transfer coefficient $h_{f}$. The induced magnetic field due to the motion of the electrically conducting fluid is negligible. This assumption is valid for small magnetic Reynolds numbers. It is also assumed that the external

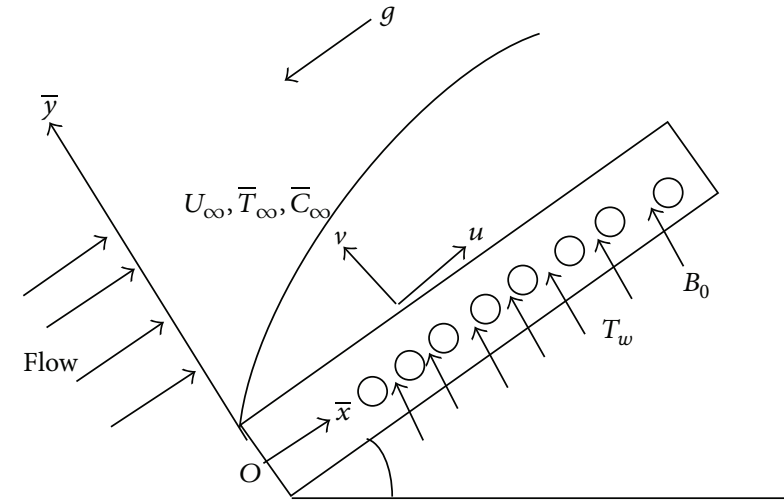

FIgURE 1: Physical configuration and the coordinate system.

electric field is zero and the electric field due to polarization of charges is negligible. It is also assumed that the pressure gradient and viscous and electrical dissipation are neglected. The physical configuration and schematic of the problem are shown in Figure 1. It is known that this is a type of FalknerSkan flow.

Furthermore the following assumptions are considered: (i) fluid has constant kinematic viscosity and thermal diffusivity and the Boussinesq approximation may be adopted for steady laminar flow, (ii) the particle diffusivity is constant, (iii) the concentration of particles is sufficiently dilute that particle coagulation in the boundary layer is negligible, and (iv) the magnetic Reynolds number is small so that the induced magnetic field is negligible in comparison with the applied magnetic field. Under these assumptions the governing Prandtl boundary-layer equations in dimensional form are as follows (see Kays et al. [20] and White [21]):

$$
\begin{gathered}
\frac{\partial \bar{u}}{\partial \bar{x}}+\frac{\partial \bar{v}}{\partial \bar{y}}=0 \\
\bar{u} \frac{\partial \bar{u}}{\partial \bar{x}}+\bar{v} \frac{\partial \bar{u}}{\partial \bar{y}}=\bar{u}_{e} \frac{d \bar{u}_{e}}{d \bar{x}}+\frac{1}{\rho_{\infty}} \frac{\partial}{\partial \bar{y}}\left[\mu(T) \frac{\partial \bar{u}}{\partial \bar{y}}\right] \\
+g \beta_{T}\left(T-T_{\infty}\right)+g \beta_{C}\left(C-C_{\infty}\right) \\
-\frac{\sigma B_{0}^{2}}{\rho}\left(\bar{u}-\bar{u}_{e}\right) \\
\bar{u} \frac{\partial T}{\partial \bar{x}}+\bar{v} \frac{\partial T}{\partial \bar{y}}=\frac{1}{\rho_{\infty} c_{p}} \frac{\partial}{\partial \bar{y}}\left[\kappa(T) \frac{\partial T}{\partial \bar{y}}\right] \\
\bar{u} \frac{\partial C}{\partial \bar{x}}+\bar{v} \frac{\partial C}{\partial \bar{y}}=\frac{\partial}{\partial \bar{y}}\left(D(C) \frac{\partial C}{\partial \bar{y}}\right)
\end{gathered}
$$

where $\bar{u}$ and $\bar{v}$ are the velocities in the $\bar{x}$ and $\bar{y}$ directions, respectively, $T$ is the temperature within the boundary layer, $T_{\infty}$ is the temperature far away from the plate, $C$ is the species concentration, $g$ is the acceleration due to gravity, $\beta_{T}$ is the volumetric coefficient of thermal expansion, $\beta_{C}$ is the volumetric coefficient of concentration expansion, $\alpha$ is 
the thermal conductivity, and $D$ is the molecular diffusivity. The respective boundary conditions are

$$
\begin{array}{r}
\bar{v}=0, \quad \bar{u}=N_{1} v \frac{\partial \bar{u}}{\partial \bar{y}}, \quad T=T_{w}+D_{1} \frac{\partial T}{\partial \bar{y}}, \\
C=C_{w} \quad \text { at } \bar{y}=0, \quad \bar{u} \longrightarrow \bar{u}_{e}, \quad T \longrightarrow T_{\infty}, \\
C \longrightarrow C_{\infty} \quad \text { as } \quad \bar{y} \longrightarrow \infty,
\end{array}
$$

where $\bar{u}_{e}$ is the velocity over the plate that should be in the form $\bar{u}_{e}=c x^{m}$. This condition will be imposed later. $\mu(T)$, $\kappa(T)$, and $D(C)$ are variable viscosity, thermal conductivity, and molecular diffusivity, respectively; the dimensions of $N_{1}$ are (velocity) ${ }^{-1}$ and the dimension of $D_{1}$ is length. It is assumed that the temperature dependent viscosity and thermal conductivity vary linearly and are given by (see Seddeek and Salem [22])

$$
\begin{aligned}
& \mu(T)=\mu_{\infty}\left[1+b_{1}\left(T_{f}-T\right)\right], \\
& \kappa(T)=\kappa_{\infty}\left[1+c\left(T-T_{\infty}\right)\right],
\end{aligned}
$$

where $\mu_{\infty}$ and $\kappa_{\infty}$ are the constant undisturbed viscosity and undisturbed thermal conductivity, $b_{1}$ is a constant with $b_{1}>0$, and $c$ is a constant which depends on the fluid. It is assumed that the concentration diffusivity varies linearly and is given by (see Seddeek and Salem [22])

$$
D(C)=D_{m}\left[1+c\left(C-C_{\infty}\right)\right]=D_{m}\left[1+D_{c} \phi\right],
$$

where $D_{m}$ is the constant concentration diffusivity.

The following dimensionless variables are introduced:

$$
\begin{aligned}
x=\frac{\bar{x}}{L}, \quad y & =\frac{\bar{y}}{L} \operatorname{Re}^{1 / 2}, \quad u=\frac{\bar{u}}{U_{\infty}}, \quad v=\frac{\bar{v}}{U_{\infty}} \operatorname{Re}^{1 / 2}, \\
\theta & =\frac{T-T_{\infty}}{T_{f}-T_{\infty}}, \quad \phi=\frac{C-C_{\infty}}{C_{w}-C_{\infty}},
\end{aligned}
$$

where $\operatorname{Re}$ is the Reynolds number, $L$ is the characteristic length, $\theta$ is the dimensionless temperature variable, and $\phi$ is the dimensionless concentration variable. Introducing the stream function $\psi$ such that $u=\partial \psi / \partial y$ and $v=-\partial \psi / \partial x$, continuity equation (1) is satisfied identically and (2)-(4) now yield

$$
\begin{aligned}
\Delta_{1} \equiv & \frac{\partial \psi}{\partial y} \frac{\partial^{2} \psi}{\partial x \partial y}-\frac{\partial \psi}{\partial x} \frac{\partial^{2} \psi}{\partial y^{2}}-u_{e} \frac{d u_{e}}{d \bar{x}}-(a+A(1-\theta)) \frac{\partial^{3} \psi}{\partial y^{3}} \\
& +A \frac{\partial^{2} \psi}{\partial y^{2}} \frac{\partial \theta}{\partial y}-\frac{g\left(T_{f}-T_{\infty}\right) L}{U_{\infty}^{2}} \beta_{T} \theta \\
& -\frac{g\left(C_{w}-C_{\infty}\right) L}{U_{\infty}^{2}} \beta_{C} \phi+M\left(\frac{\partial \psi}{\partial y}-u_{e}\right)=0,
\end{aligned}
$$

$$
\begin{aligned}
\Delta_{2} & \equiv \frac{\partial \psi}{\partial y} \frac{\partial \theta}{\partial x}-\frac{\partial \psi}{\partial x} \frac{\partial \theta}{\partial y}-\frac{1}{\operatorname{Pr}}[1+S \theta] \frac{\partial^{2} \theta}{\partial y^{2}}-\frac{1}{\operatorname{Pr}} S\left(\frac{\partial \theta}{\partial y}\right)^{2} \\
= & 0 \\
\Delta_{3} \equiv & \frac{\partial \psi}{\partial y} \frac{\partial \phi}{\partial x}-\frac{\partial \psi}{\partial x} \frac{\partial \phi}{\partial y}-\frac{1}{\mathrm{Sc}}\left[1+D_{c} \phi\right] \frac{\partial^{2} \phi}{\partial y^{2}} \\
& -\frac{1}{\mathrm{Sc}} D_{c}\left(\frac{\partial \phi}{\partial y}\right)^{2}=0 .
\end{aligned}
$$

The boundary conditions are

$$
\begin{gathered}
\frac{\partial \psi}{\partial x}=0, \quad \frac{\partial \psi}{\partial y}=a \frac{\partial^{2} \psi}{\partial y^{2}}, \quad \theta=1+b \frac{\partial \theta}{\partial y}, \\
\phi=1 \quad \text { at } y=0, \\
\frac{\partial \psi}{\partial y} \longrightarrow u_{e}(x), \quad \theta \longrightarrow 0, \quad \phi \longrightarrow 0 \quad \text { as } y \longrightarrow \infty .
\end{gathered}
$$

In the above equations the parameters are defined as

$$
\begin{gathered}
\operatorname{Re}=\frac{U_{\infty} L}{v}, \quad \mathrm{Sc}=\frac{v}{D}, \quad M=\frac{\sigma B_{0}^{2} L}{\rho U_{\infty}}, \\
\operatorname{Pr}=\frac{\mu c_{p}}{\kappa}, \quad A=b_{1}\left(T_{f}-T_{\infty}\right), \\
S=c\left(T_{f}-T_{w}\right), \quad a=\frac{N_{1} v}{L} \sqrt{\mathrm{Re},} \\
b=\frac{D_{1} \sqrt{\mathrm{Re}}}{L}, \quad D_{c}=c\left(C_{w}-C_{\infty}\right),
\end{gathered}
$$

where Re is the Reynolds number, Sc is the Schmidt number, $M$ is the magnetic parameter, $\mathrm{Pr}$ is the Prandtl number of the fluid, $S$ is the thermal conductivity parameter, $A$ is the viscosity parameter, $D_{c}$ is the concentration diffusivity parameter, $a$ is the velocity slip parameter, and $b$ is the thermal slip parameter.

\section{Application of Group Transformations}

Determining similarity solutions of (9)-(10) is equivalent to determining invariant solutions of these equations under a particular continuous one parameter group (Hamad et al. [23] and Kandasamy et al. [24]). Thus we search for a transformation group from the elementary set of oneparameter scaling transformations as one of the techniques that are defined by the following group which is called G1:

$$
\begin{aligned}
\mathrm{G} 1: x^{*} & =x e^{\varepsilon \alpha_{1}}, & y^{*}=y e^{\varepsilon \alpha_{2}}, & \psi^{*}=\psi e^{\varepsilon \alpha_{3},} \\
\theta^{*} & =\theta e^{\varepsilon \alpha_{4}}, & \phi^{*}=\phi e^{\varepsilon \alpha_{5}}, & \beta_{T}^{*}=\beta_{T} e^{\varepsilon \alpha_{6}}, \\
\beta_{\mathrm{C}}^{*} & =\beta_{C} e^{\varepsilon \alpha_{7}}, & u_{e}^{*}=u_{e} e^{\varepsilon \alpha_{8}} . &
\end{aligned}
$$

Here $\varepsilon(\neq 0)$ is a parameter of the group and the $\alpha$ 's are arbitrary real numbers whose connection will be determined 
by our analysis. The transformations listed in (12) may be treated as point transformations which transform the coordinates

$$
\left(x, y, \psi, \theta, \phi, \beta_{T}, \beta_{C}, u_{e}\right) \text { to }\left(x^{*}, y^{*}, \psi^{*}, \theta^{*}, \phi^{*}, \beta_{T}^{*}, \beta_{C}^{*}, u_{e}^{*}\right) .
$$

The system (9)-(10) remains invariant under the group transformation G1. Hence we have the following relationships among the parameters, namely,

$$
\begin{gathered}
2 \alpha_{3}-\alpha_{1}-2 \alpha_{2}=2 \alpha_{8}-\alpha_{1}=\alpha_{3}-3 \alpha_{2} \\
=\alpha_{3}-\alpha_{2}=\alpha_{3}+\alpha_{4}-3 \alpha_{2}=\alpha_{8} \\
=\alpha_{4}+\alpha_{6}=\alpha_{5}+\alpha_{7} \\
\alpha_{3}+\alpha_{4}-\alpha_{1}-\alpha_{2}=\alpha_{4}-2 \alpha_{2}=2 \alpha_{4}-2 \alpha_{2}, \\
\alpha_{3}+\alpha_{5}-\alpha_{1}-\alpha_{2}=\alpha_{5}-2 \alpha_{2}=2 \alpha_{5}-2 \alpha_{2} .
\end{gathered}
$$

From boundary conditions (10), these will be invariant if

$$
\begin{gathered}
\alpha_{2}-\alpha_{3}=2 \alpha_{2}-\alpha_{3}, \quad-\alpha_{4}=\alpha_{2}-\alpha_{4}, \\
\alpha_{5}=0, \quad \alpha_{2}-\alpha_{3}=-\alpha_{8} .
\end{gathered}
$$

Solving (14) and (15), we obtain

$$
\alpha_{2}=\alpha_{4}=\alpha_{5}=0, \quad \alpha_{1}=\alpha_{3}=\alpha_{6}=\alpha_{7}=\alpha_{8} .
$$

With these relations the boundary conditions remain invariant.

The set of transformations G1 in (12) then reduces to

$$
\begin{gathered}
x^{*}=x e^{\varepsilon \alpha_{1}}, \quad y^{*}=y, \quad \psi^{*}=\psi e^{\varepsilon \alpha_{1}}, \\
\theta^{*}=\theta, \quad \phi^{*}=\phi, \quad \beta_{T}^{*}=\beta_{T} e^{\varepsilon \alpha_{1}}, \quad \beta_{C}^{*}=\beta_{C} e^{\varepsilon \alpha_{1}}, \\
u_{e}^{*}=u_{e} e^{\varepsilon \alpha_{1}} .
\end{gathered}
$$

Using a Taylor series expansion in powers of $\varepsilon$, retaining terms up to first order, and neglecting higher powers of $\varepsilon$ results in

$$
\begin{aligned}
& x^{*}-x=\varepsilon \alpha_{1} x, \quad y^{*}-y=0, \\
& \psi^{*}-\psi=\varepsilon \alpha_{1} \psi, \quad \theta^{*}-\theta=0, \\
& \phi^{*}-\phi=0, \quad \beta_{T}^{*}-\beta_{T}=\varepsilon \alpha_{1} \beta_{T}, \\
& \beta_{C}^{*}-\beta_{C}=\varepsilon \alpha_{1} \beta_{C}, \quad u_{e}^{*}-u_{e}=\varepsilon \alpha_{1} u_{e} .
\end{aligned}
$$

The characteristic equations are

$\frac{d x}{\alpha_{1} x}=\frac{d y}{0}=\frac{d \psi}{\alpha_{1} \psi}=\frac{d \theta}{0}=\frac{d \phi}{0}=\frac{d \beta_{T}}{\alpha_{1} \beta_{T}}=\frac{d \beta_{C}}{\alpha_{1} \beta_{C}}=\frac{d u_{e}}{\alpha_{1} u_{e}}$.

Solving the above characteristic equations gives

$$
\begin{array}{ccc}
\eta=y, & \psi=x f(\eta), & \theta=\theta(\eta), \\
\phi=\phi(\eta), & \beta_{T}=\beta_{T_{0}} x, & \beta_{C}=\beta_{C_{0}} x, \\
& u_{e}=U_{\infty} x .
\end{array}
$$

Substituting (20) into (9)-(10) yields

$$
\begin{gathered}
{[1+A(1-\theta)] f^{\prime \prime \prime}+\left(f-A \theta^{\prime}\right) f^{\prime \prime}-f^{\prime 2}} \\
-M\left(f^{\prime}-1\right)+1+\operatorname{Gr} \theta+\operatorname{Gc} \phi=0, \\
{[1+S \theta] \theta^{\prime \prime}+S \theta^{\prime 2}+\operatorname{Pr} f \theta^{\prime}=0,} \\
{\left[1+D_{c} \phi\right] \phi^{\prime \prime}+D_{c} \phi^{\prime 2}+\operatorname{Sc} f \phi^{\prime}=0 .}
\end{gathered}
$$

Here $\mathrm{Gr}=g\left(T_{f}-T_{\infty}\right) L \beta_{T_{0}} / U_{\infty}^{2}$ and $\mathrm{Gc}=g\left(C_{w}-\right.$ $\left.C_{\infty}\right) L \beta_{c_{0}} / U_{\infty}^{2}$ are Grashof numbers based on temperature and on concentration, respectively.

The corresponding boundary conditions are

$$
\begin{gathered}
f(0)=0, \quad f^{\prime}(0)=a f^{\prime \prime}(0), \\
\theta(0)=1+b \theta^{\prime}(0), \quad \phi(0)=1, \\
f^{\prime}(\infty) \longrightarrow 1, \quad \theta(\infty) \longrightarrow 0, \quad \phi(\infty) \longrightarrow 0 .
\end{gathered}
$$

To obtain a similarity solution for the energy equation, the quantity $b$ must be independent of $\bar{x}$ and for this to occur the heat transfer coefficient $h_{f}$ must be directly proportional to $(\bar{x})^{-1 / 2}$.

3.1. Parameters of Physical Interest. We are interested in the friction factor $\mathrm{C}_{f}$, Nusselt number $\mathrm{Nu}$, and Sherwood number Sh, respectively. Physically, $C_{f}$ indicates wall shear stress and $\mathrm{Nu}$ indicates the rate of heat transfer whilst Sh indicates the rate of mass transfer. These quantities may be conveniently determined from

$$
\begin{gathered}
C_{f}=\frac{\mu}{\rho U_{\infty}^{2}}\left(\frac{\partial \bar{u}}{\partial \bar{y}}\right)_{\bar{y}=0}, \quad \mathrm{Nu}=\frac{-\bar{x}}{T_{w}-T_{\infty}}\left(\frac{\partial T}{\partial \bar{y}}\right)_{\bar{y}=0}, \\
\mathrm{Sh}=\frac{-\bar{x}}{C_{w}-C_{\infty}}\left(\frac{\partial C}{\partial \bar{y}}\right)_{\bar{y}=0} .
\end{gathered}
$$

By substituting (10) and (19) into (23), we obtain

$$
\begin{gathered}
\operatorname{Re}^{1 / 2} C_{f}=[1+A(1-\theta(0))] f^{\prime \prime}(0), \\
\operatorname{Re}^{-1 / 2} \mathrm{Nu}=-\theta^{\prime}(0), \\
\operatorname{Re}^{-1 / 2} \mathrm{Sh}=-\phi^{\prime}(0) .
\end{gathered}
$$

From (24) it can be shown that the skin friction factor $C_{f}$, the Nusselt number $\mathrm{Nu}$, and the Sherwood number Sh are proportional to the numerical values $f^{\prime \prime}(0),-\theta^{\prime}(0)$, and $-\phi^{\prime}(0)$, respectively.

\section{The Keller Box Method}

Equation (21) subject to boundary conditions (22) is solved numerically using a very efficient finite difference scheme known as the Keller Box method. The details of this method are described in Cebeci and Bradshaw [25] and $\mathrm{Na}$ [26]. For more information refer to Keller [27, 28]. 


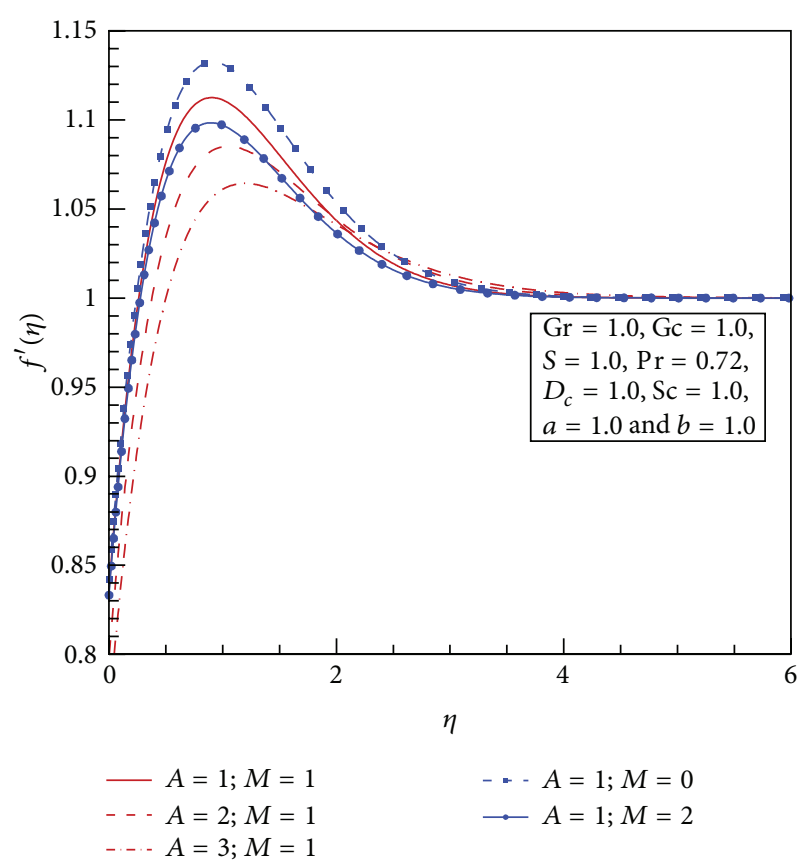

FIGURE 2: Effects of the viscosity parameter $A$ and the magnetic parameter $M$ on the dimensionless velocity.

\section{Results and Discussion}

Applying scaling group transformations to analyze the governing equations and the boundary conditions, the two independent variables are reduced by one. Consequently the governing equations reduce to a system of nonlinear ordinary differential equations with the appropriate boundary conditions. The transformed momentum, energy, and concentration equation (21) subject to the boundary conditions (22) were solved numerically by using the Keller Box method. We obtained velocity, temperature, and concentration profile graphs for different values of governing parameters.

Figures 2, 3, and 4 show the effects of the viscosity parameter and the magnetic parameter on the velocity, temperature, and concentration distributions, respectively. The velocity distribution decreases with increasing $A$ and $M$ whereas they have no significant effect on the temperature and concentration distributions. This behavior can be predicted from (21) and also the physical definition of parameters $A$ and $M$, since the viscosity and magnetic parameters only appear in the momentum equation. Figures 5, 6, and 7 show the effect of the thermal and mass Grashof numbers on the velocity, temperature, and concentration distributions, respectively. Physically, since the thermal Grashof number (Gr) is the ratio of buoyancy to viscous forces in the boundary layer, increasing its value suggests an increase in the buoyancy forces relative to the viscous forces and this is clearly reflected in the progressive increase in the velocity of the flow. Increase in the mass transfer Grashof number (Gc) yields a similar effect on the velocity of the flow. Moreover, the reverse trend is seen for the temperature and concentration distributions.

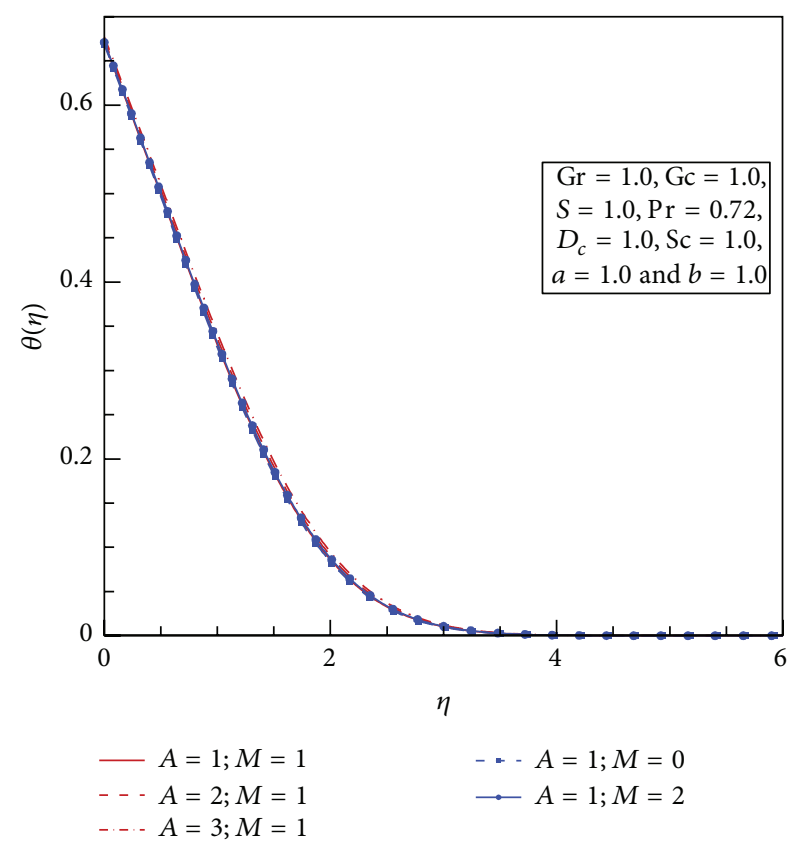

FIGURE 3: Effects of the viscosity parameter $A$ and the magnetic parameter $M$ on the dimensionless temperature.

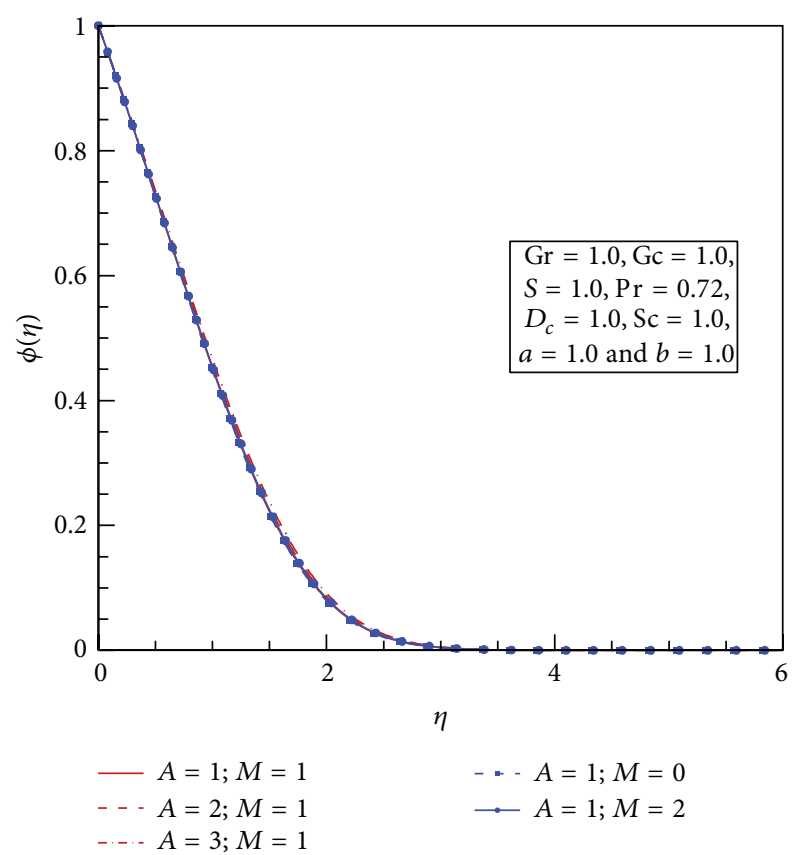

FIGURE 4: Effects of the viscosity parameter $A$ and the magnetic parameter $M$ on the dimensionless concentration.

Figures 8, 9, and 10 illustrate the influence of the thermal conductivity parameter $S$ and Prandtl number $\operatorname{Pr}$ on the velocity, temperature, and concentration distributions, respectively. It is observed that the velocity and temperature distributions increase with increasing thermal conductivity parameter and decrease with increasing Prandtl number. 


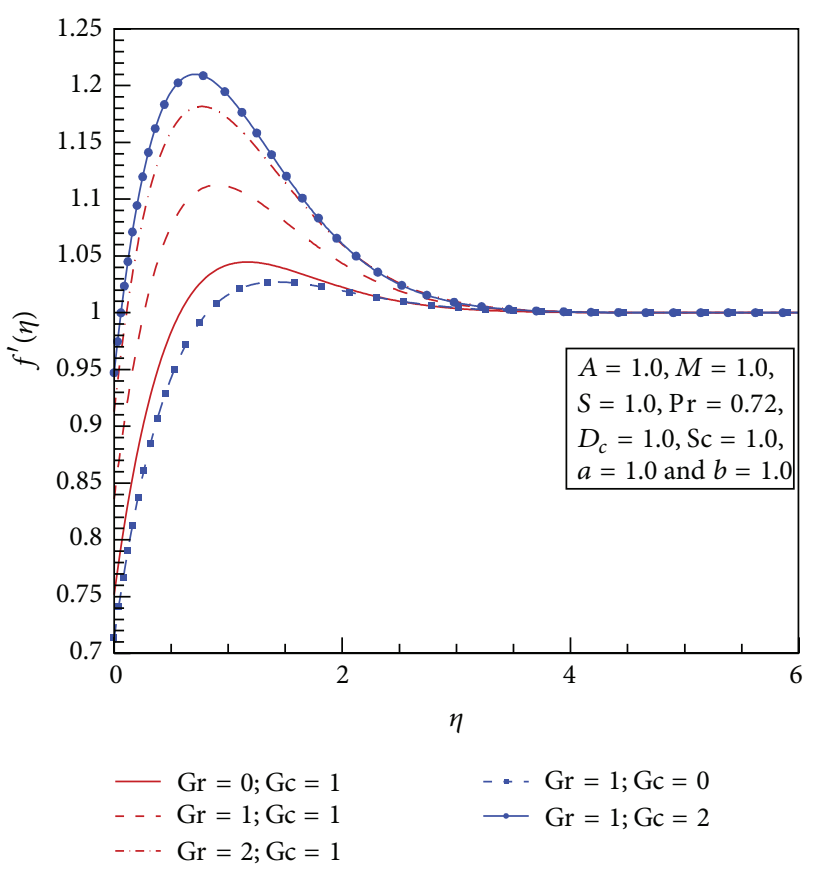

FIGURE 5: Effects of the thermal Grashof number Gr and the mass Grashof number Gc on the dimensionless velocity.

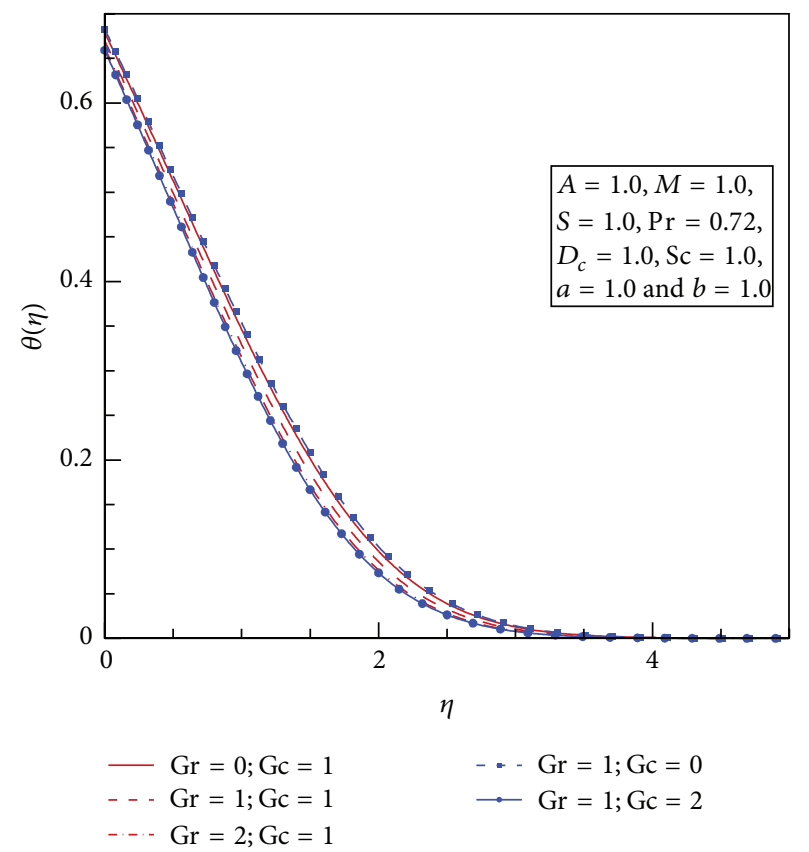

FIGURE 6: Effects of the thermal Grashof number Gr and the mass Grashof number Gc on the dimensionless temperature.

This is in agreement physically since the thermal boundarylayer thickness decreases with increasing Pr. The thermal conductivity parameter $S$ and the Prandtl number Pr have no significant effect on the concentration distribution and this can be predicted from (21). The effects of the concentration diffusivity parameter $D_{c}$ and the Schmidt number Sc on

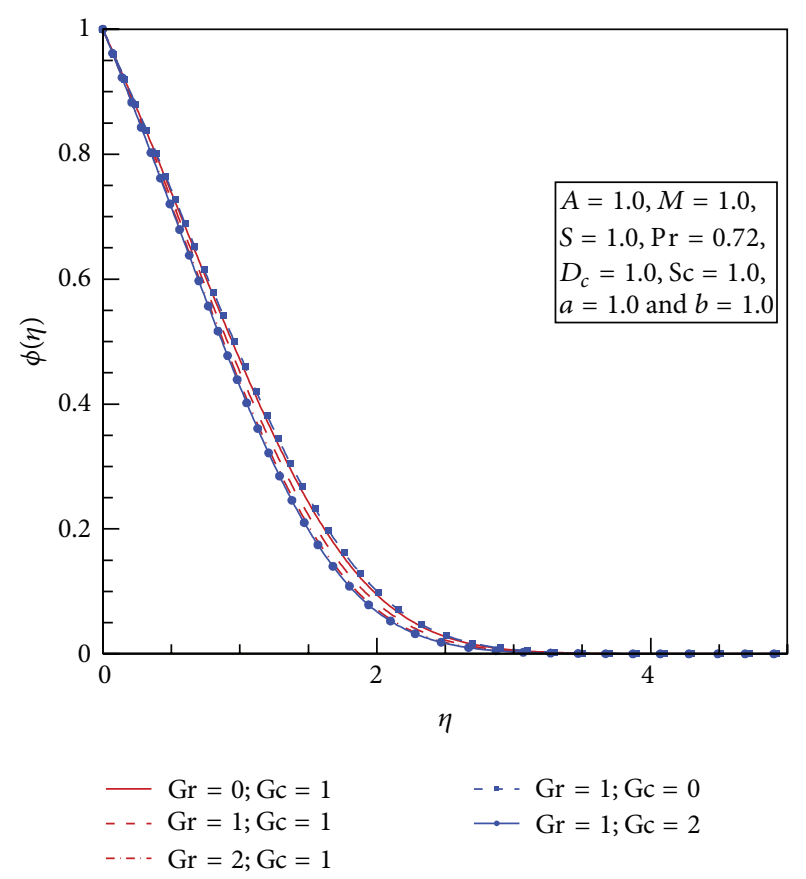

FIGURE 7: Effects of the thermal Grashof number Gr and the mass Grashof number $\mathrm{Gc}$ on the dimensionless concentration.

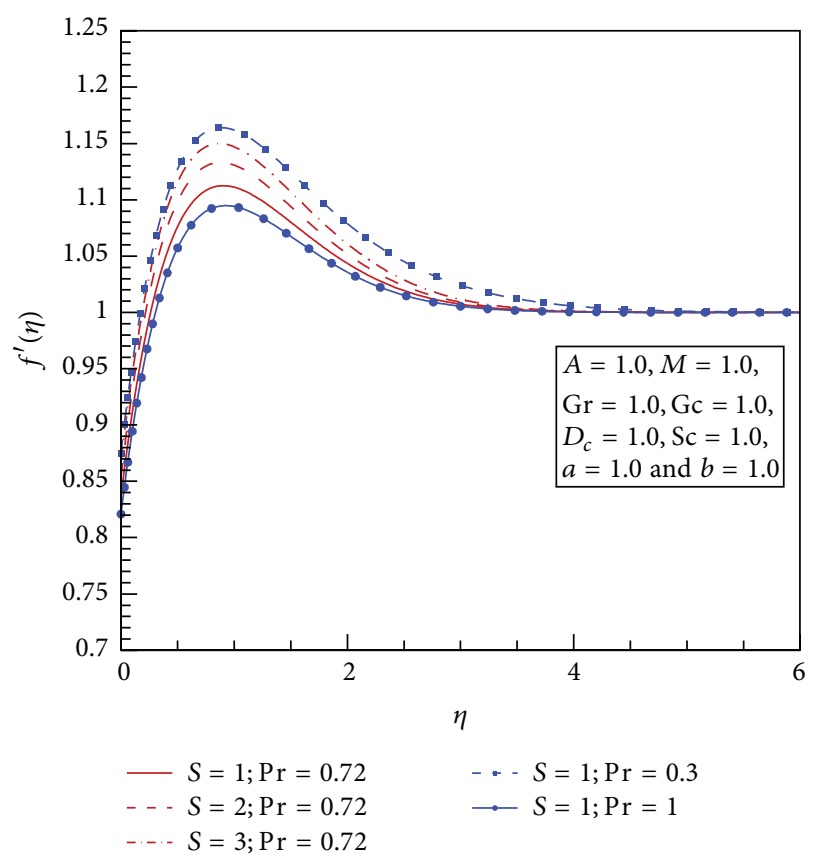

FIGURE 8: Effects of the thermal conductivity parameter $S$ and the Prandtl number Pr on the dimensionless velocity.

the velocity, temperature, and concentration distributions are shown in Figures 11-13. The velocity and concentration distributions increase with increasing concentration diffusivity parameter whereas they decrease with increasing Schmidt number. Since Schmidt number is the ratio of viscosity to diffusivity, this behavior can be predicted. From Figure 12, 


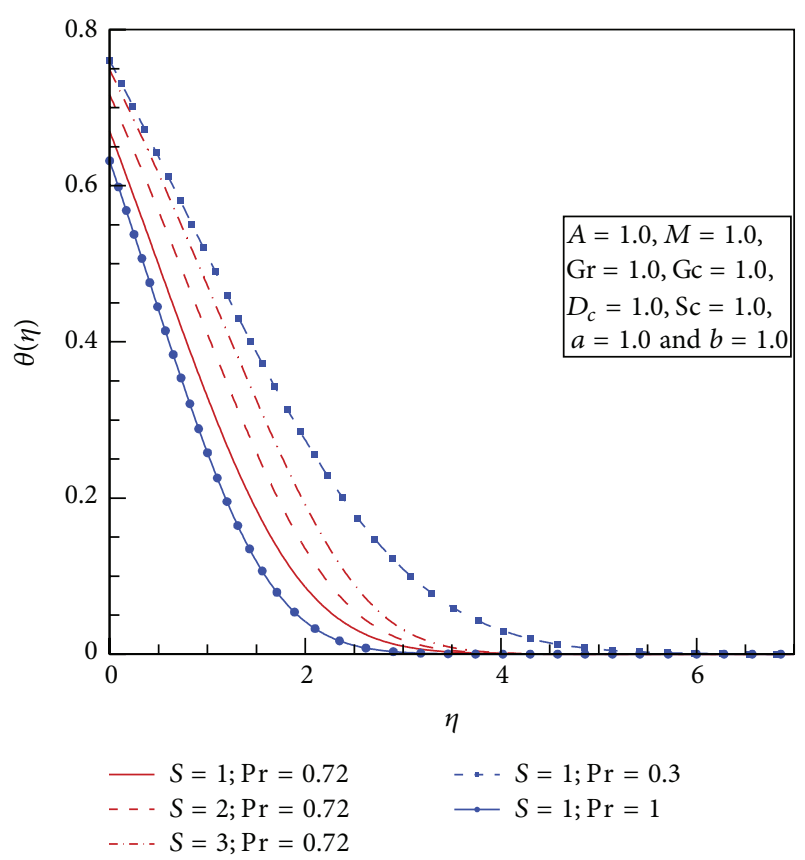

FIGURE 9: Effects of the thermal conductivity parameter $S$ and the Prandtl number Pr on the dimensionless temperature.

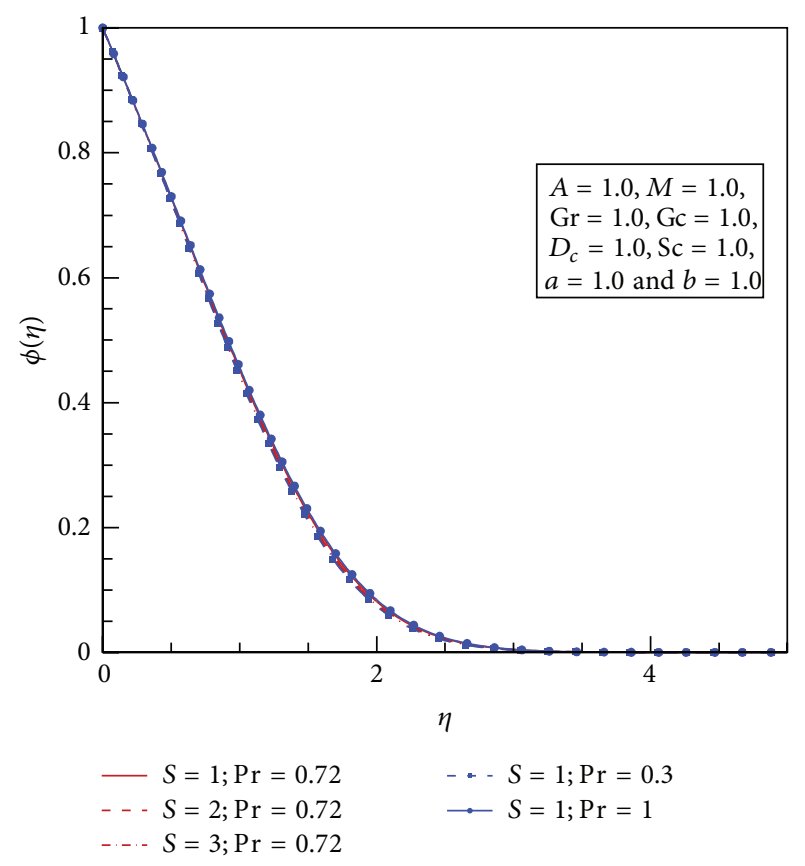

FIgURE 10: Effects of the thermal conductivity parameter $S$ and the Prandtl number Pr on the dimensionless concentration.

the concentration diffusivity parameter and the Schmidt number have no significant effect on the temperature distribution. In Figures 14-16 effects of the velocity slip parameter $(a)$ and the thermal slip parameter $(b)$ are depicted. In Figure 14 it is observed that velocity distribution increases with increasing velocity slip parameter and decreases with increasing thermal slip parameter. From Figure 15 we observe

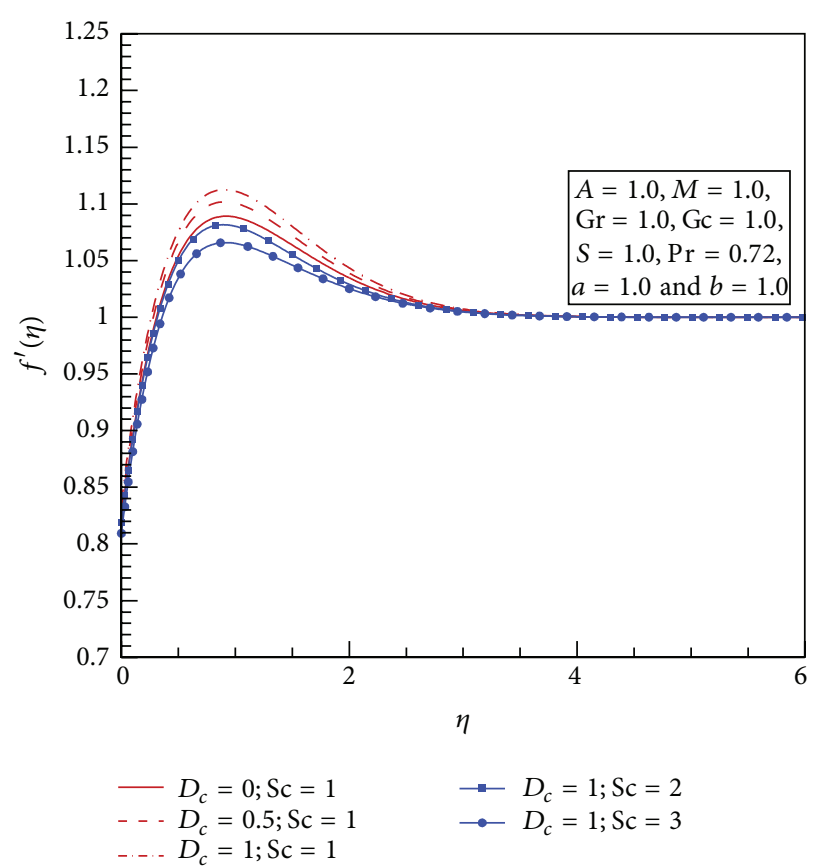

FIGURE 11: Effects of the concentration diffusivity parameter $D_{c}$ and the Schmidt number Sc on the dimensionless velocity.

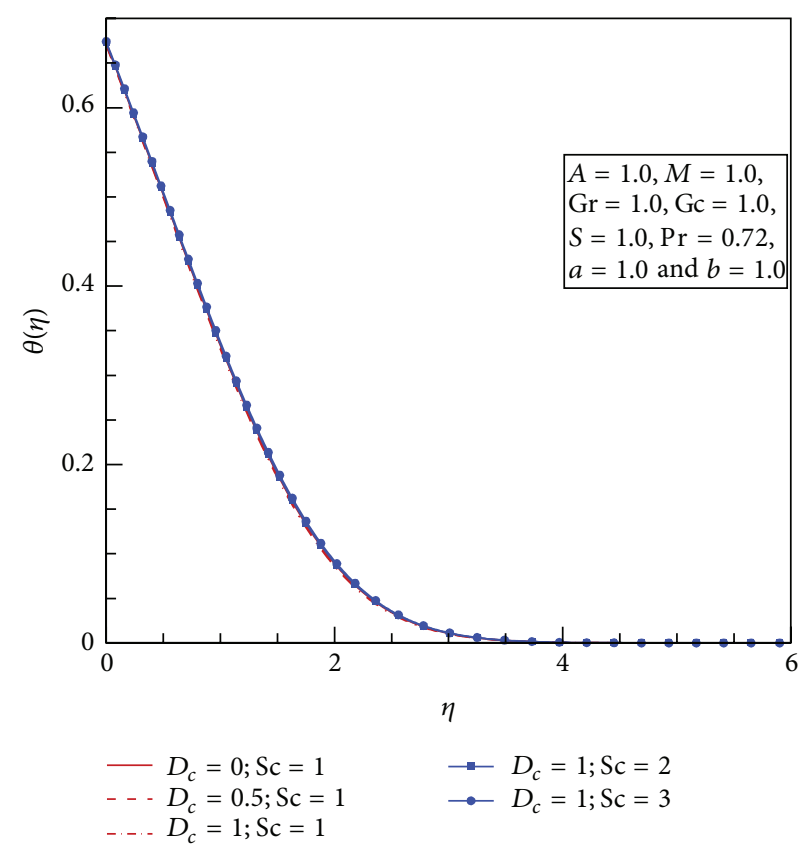

FIGURE 12: Effects of the concentration diffusivity parameter $D_{c}$ and the Schmidt number Sc on the dimensionless temperature.

that as the velocity slip parameter and the thermal slip parameter increase the temperature distribution decreases. Figure 16 shows that the concentration distribution decreases with increasing velocity slip parameter and increases with increasing thermal slip parameter. In some of the velocity profiles an overshoot of the velocity profile is observed. This depends on the boundary conditions. In other words, since 


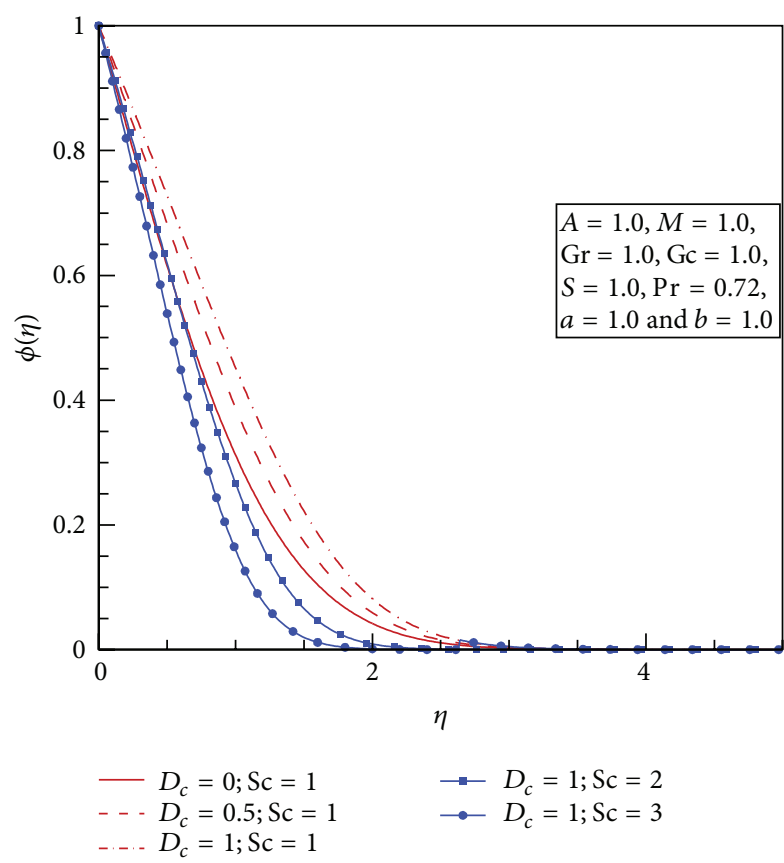

FIGURE 13: Effects of the concentration diffusivity parameter $D_{c}$ and the Schmidt number Sc on the dimensionless concentration.

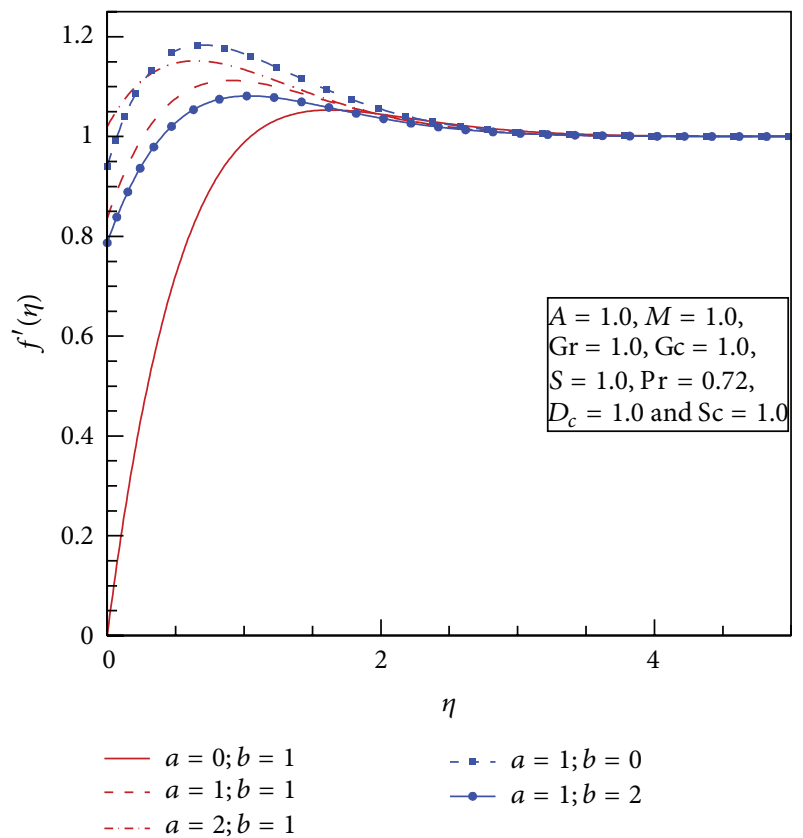

FIGURE 14: Effects of the velocity slip parameter $a$ and the thermal slip parameter $b$ on the dimensionless velocity.

there is not a no-slip condition on the plate, a larger velocity rather than free stream velocity can exist inside the boundary layer. With attention to boundary conditions (5) an overshoot of the velocity is likely.

In Table 1 choosing $A=1.0, S=1.0, D_{c}=1.0, \mathrm{Sc}=1.0$; $a=1.0$, and $b=1.0$, numerical values of $f^{\prime \prime}(0), \theta^{\prime}(0)$, and

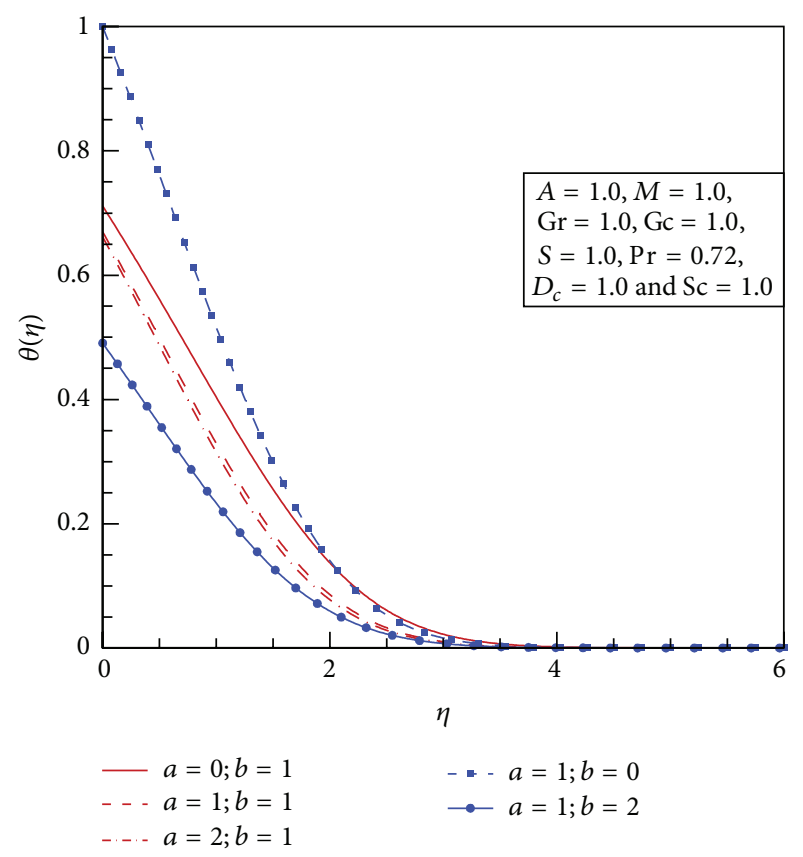

FIGURE 15: Effects of the velocity slip parameter $a$ and the thermal slip parameter $b$ on the dimensionless temperature.

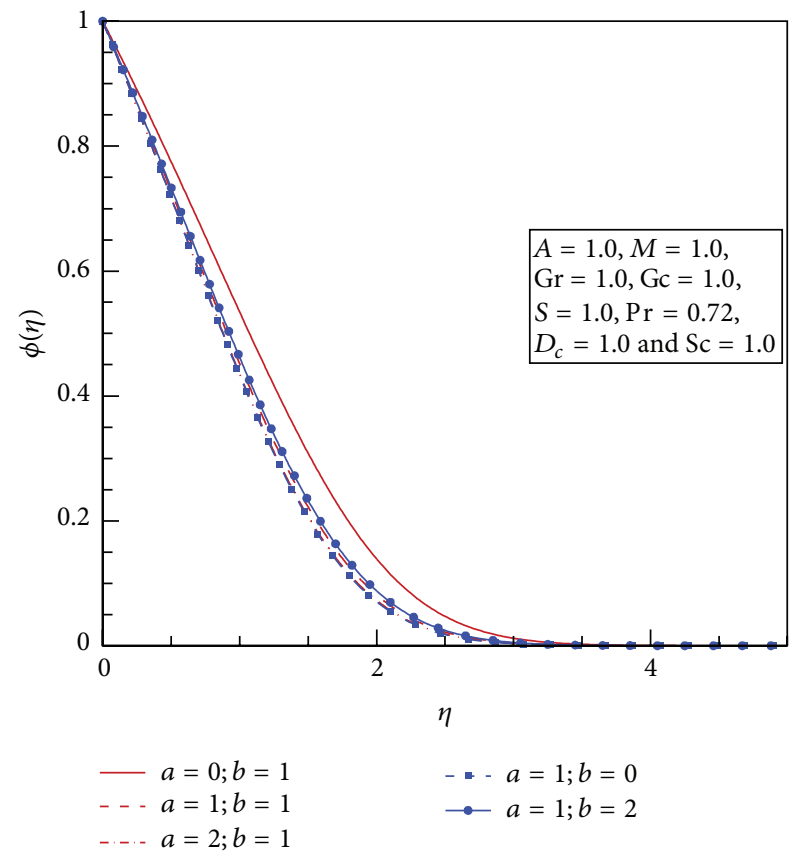

FIGURE 16: Effects of the velocity slip parameter $a$ and the thermal slip parameter $b$ on the dimensionless concentration.

$\varphi^{\prime}(0)$ are shown for different values of the parameters $\mathrm{Gr}, \mathrm{Gc}$, $M$, and Pr. Results of Figures 2-16 are verified.

\section{Conclusions}

A numerical study based on the Keller Box method for MHD heat and mass transfer flow of a steady viscous 
TABLE 1: Numerical results of $f^{\prime \prime}(0), \theta^{\prime}(0)$, and $\varphi^{\prime}(0)$ for different values of the parameters $\mathrm{Gr}, \mathrm{Gc}, M$, and $\operatorname{Pr}$ when $A=1.0, S=1.0$, $D_{c}=1.0, \mathrm{Sc}=1.0 ; a=1.0$, and $b=1.0$.

\begin{tabular}{lcccccc}
\hline Gr & Gc & $M$ & $\operatorname{Pr}$ & $f^{\prime \prime}(0)$ & $-\theta^{\prime}(0)$ & $-\varphi^{\prime}(0)$ \\
\hline 1 & 1 & 1 & 0.72 & 0.835798 & 0.33 & 0.51803 \\
0 & 1 & 1 & 0.72 & 0.751505 & 0.32094 & 0.49805 \\
2 & 1 & 1 & 0.72 & 0.912888 & 0.33783 & 0.53563 \\
1 & 0 & 1 & 0.72 & 0.713589 & 0.31708 & 0.48958 \\
1 & 2 & 1 & 0.72 & 0.947207 & 0.34088 & 0.54264 \\
1 & 1 & 0 & 0.72 & 0.842129 & 0.33159 & 0.52146 \\
1 & 1 & 2 & 0.72 & 0.833216 & 0.32895 & 0.51581 \\
1 & 1 & 1 & 0.3 & 0.87512 & 0.23957 & 0.52956 \\
1 & 1 & 1 & 1 & 0.820823 & 0.36801 & 0.51389 \\
\hline
\end{tabular}

incompressible fluid over a flat plate has been performed. We have investigated the effects of various governing parameters, namely, the viscosity parameter $A$, the magnetic field $M$, thermal Grashof number Gr, mass transfer Grashof number Gc, thermal conductivity parameter $S$, Prandtl number Pr, concentration diffusivity parameter $D_{c}$, Schmidt number Sc, velocity slip parameter $a$, and thermal slip parameter $b$ on flow field and heat transfer characteristics. The following conclusions can be made.

(1) The thickness of the velocity boundary layer decreases with an increase in viscosity parameter $A$, magnetic field $M$, Schmidt number Sc, and thermal slip parameter $b$.

(2) The thickness of the velocity boundary layer increases with an increase in thermal Grashof number Gr, mass transfer Grashof number Gc, thermal conductivity parameter $S$, concentration diffusivity parameter $D_{c}$, and velocity slip parameter $a$.

(3) The thickness of the thermal boundary layer decreases with an increase in thermal Grashof number Gr, mass transfer Grashof number Gc, Prandtl number Pr, velocity slip parameter $a$, and thermal slip parameter $b$.

(4) The thickness of the thermal boundary layer increases with an increase in thermal conductivity parameter $S$.

(5) The thickness of the concentration boundary layer decreases with an increase in thermal Grashof number Gr, mass transfer Grashof number Gc, Schmidt number Sc, and velocity slip parameter $a$.

(6) The thickness of the concentration boundary layer increases with an increase in concentration diffusivity parameter $D_{c}$ and thermal slip parameter $b$.

\section{Nomenclature}

A: Viscosity parameter

a: Velocity slip parameter

$B_{0}$ : Strength of magnetic field

$b$ : Thermal slip parameter

$b_{1}$ : Constant
C: Concentration

c: Constant

$C_{f}$ : Friction factor

$D$ : Molecular diffusivity

$D_{c}$ : Concentration diffusivity parameter

$D_{m}$ : Constant concentration diffusivity

$f:$ Dimensionless velocity functions

$g$ : Gravitation acceleration

Gc: Grashof number based on temperature

Gr: Grashof number based on concentration

$h$ : Heat transfer coefficient

L: $\quad$ Characteristic length

M: Magnetic parameter

$\mathrm{Nu}$ : Nusselt number

Pr: Prandtl number

Re: Reynolds number

S: Thermal conductivity parameter

Sc: Schmidt number

Sh: Sherwood number

T: Temperature

$\bar{u}: \quad$ Velocity in $\bar{x}$-direction

$\bar{u}_{e}:$ Velocity over the plate

$\bar{v}$ : Velocity in $\bar{y}$-direction

$\bar{x}$ : Distance along the plate

$\bar{y}$ : Distance normal to the plate.

\section{Greek Letters}

$\alpha$ : Thermal conductivity

$\beta_{C}$ : Volumetric coefficient of concentration expansion

$\beta_{T}$ : Volumetric coefficient of thermal expansion

$\phi$ : Dimensionless concentration

$\eta$ : Similarity variable

$\mu$ : Dynamic viscosity

$\theta$ : Dimensionless temperature

$\kappa$ : Thermal conductivity

$\rho$ : Density of fluid

$\psi$ : Stream function.

Subscript and Superscript

$f$ : Fluid

$w$ : Plate

$\infty$ : Conditions far away from the plate

': Differentiation with respect to $\eta$.

\section{Conflict of Interests}

The authors declare that there is no conflict of interests regarding the publication of this paper.

\section{Acknowledgments}

The authors thank the reviewers for suggesting certain changes to the original paper, for their valuable comments which improved the paper, and for their interest in our work. S. Abelman gratefully acknowledges the support from 
the University of the Witwatersrand, Johannesburg, and the National Research Foundation, Pretoria, South Africa.

\section{References}

[1] M. M. Rashidi, T. Hayat, and A. B. Parsa, "Solving of boundarylayer equations with transpiration effects, governance on a vertical permeable cylinder using modified differential transform method," Heat Transfer-Asian Research, vol. 40, no. 8, pp. 677692, 2011.

[2] M. Turkyilmazoglu, "MHD fluid flow and heat transfer due to a shrinking rotating disk," Computers \& Fluids, vol. 90, pp. 51-56, 2014.

[3] J. Zhang and M.-J. Ni, "A consistent and conservative scheme for MHD flows with complex boundaries on an unstructured Cartesian adaptive system," Journal of Computational Physics, vol. 256, pp. 520-542, 2014.

[4] M. M. Rashidi and E. Erfani, "Analytical method for solving steady MHD convective and slip flow due to a rotating disk with viscous dissipation and Ohmic heating," Engineering Computations, vol. 29, no. 6, pp. 562-579, 2012.

[5] S. Mukhopadhyay, G. C. Layek, and Sk. A. Samad, "Study of MHD boundary layer flow over a heated stretching sheet with variable viscosity," International Journal of Heat and Mass Transfer, vol. 48, no. 21-22, pp. 4460-4466, 2005.

[6] H. I. Andersson, "MHD flow of a viscoelastic fluid past a stretching surface," Acta Mechanica, vol. 95, no. 1-4, pp. 227230, 1992.

[7] M. M. Rashidi, N. Laraqi, and A. B. Parsa, "Analytical modeling of heat convection in magnetized micropolar fluid by using modified differential transform method," Heat Transfer-Asian Research, vol. 40, no. 3, pp. 187-204, 2011.

[8] A. B. Parsa, M. M. Rashidi, and T. Hayat, "MHD boundary-layer flow over a stretching surface with internal heat generation or absorption," Heat Transfer-Asian Research, vol. 42, no. 6, pp. 500-514, 2013.

[9] T. Watanabe and I. Pop, "Magnetohydrodynamic free convection flow over a wedge in the presence of a transverse magnetic field," International Communications in Heat and Mass Transfer, vol. 20, no. 6, pp. 871-881, 1993.

[10] M. Kumari and G. Nath, "Analytical solution of unsteady threedimensional MHD boundary layer flow and heat transfer due to impulsively stretched plane surface," Communications in Nonlinear Science and Numerical Simulation, vol. 14, no. 8, pp. 3339-3350, 2009.

[11] R. Moreau, Magnetohydrodynamics, Kluwer Academic, Dordrecht, The Netherlands, 1990.

[12] O. D. Makinde, "Thermal stability of a reactive viscous flow through a porous-saturated channel with convective boundary conditions," Applied Thermal Engineering, vol. 29, no. 8-9, pp. 1773-1777, 2009.

[13] O. D. Makinde and P. Sibanda, "Magnetohydrodynamic mixedconvective flow and heat and mass transfer past a vertical plate in a porous medium with constant wall suction," ASME Journal of Heat Transfer, vol. 130, no. 11, pp. 1-8, 2008.

[14] O. D. Makinde and A. Ogulu, "The effect of thermal radiation on the heat and mass transfer flow of a variable viscosity fluid past a vertical porous plate permeated by a transverse magnetic field," Chemical Engineering Communications, vol. 195, no. 12, pp. 1575-1584, 2008.
[15] R. Rajeswari, B. Jothiram, and V. K. Nelson, "Chemical reaction, heat and mass transfer on nonlinear MHD boundary layer flow through a vertical porous surface in the presence of suction," Applied Mathematical Sciences, vol. 3, no. 49-52, pp. 2469-2480, 2009.

[16] T.-M. Chen, "Radiation effects on magnetohydrodynamic free convection flow," Journal of Thermophysics and Heat Transfer, vol. 22, no. 1, pp. 125-128, 2008.

[17] A. Ishak, "Similarity solutions for flow and heat transfer over a permeable surface with convective boundary condition," Applied Mathematics and Computation, vol. 217, no. 2, pp. 837842, 2010.

[18] O. D. Makinde, "Similarity solution of hydromagnetic heat and mass transfer over a vertical plate with a convective surface boundary condition," International Journal of Physical Sciences, vol. 5, no. 6, pp. 700-710, 2010.

[19] M. M. Rashidi, M. Ali, N. Freidoonimehr, and F. Nazari, "Parametric analysis and optimization of entropy generation in unsteady MHD flow over a stretching rotating disk using artificial neural network and particle swarm optimization algorithm," Energy, vol. 55, pp. 497-510, 2013.

[20] W. M. Kays, M. E. Crawford, and B. Weigand, Convective Heat and Mass Transfer, McGraw-Hill, Boston, Mass, USA, 4th edition, 2005.

[21] F. M. White, Viscous Fluid Flow, McGraw-Hill, Boston, Mass, USA, 2nd edition, 1991.

[22] M. A. Seddeek and A. M. Salem, "Laminar mixed convection adjacent to vertical continuously stretching sheets with variable viscosity and variable thermal diffusivity," Heat and Mass Transfer, vol. 41, no. 12, pp. 1048-1055, 2005.

[23] M. A. A. Hamad, Md. J. Uddin, and A. I. Md. Ismail, "Investigation of combined heat and mass transfer by Lie group analysis with variable diffusivity taking into account hydrodynamic slip and thermal convective boundary conditions," International Journal of Heat and Mass Transfer, vol. 55, no. 4, pp. 1355-1362, 2012.

[24] R. Kandasamy, I. Muhaimin, and H. Bin Saim, "Lie group analysis for the effect of temperature-dependent fluid viscosity with thermophoresis and chemical reaction on MHD free convective heat and mass transfer over a porous stretching surface in the presence of heat source/sink," Communications in Nonlinear Science and Numerical Simulation, vol. 15, no. 8, pp. 2109-2123, 2010.

[25] T. Cebeci and P. Bradshaw, Physical and Computational Aspects of Convective Heat Transfer, Springer, New York, NY, USA, 1984.

[26] T. Y. Na, Computational Methods in Engineering Boundary Value Problem, Academic Press, New York, NY, USA, 1979.

[27] H. B. Keller, "Numerical methods in boundary-layer theory," Annual Review of Fluid Mechanics, vol. 10, pp. 417-433, 1978.

[28] H. B. Keller, "A new difference scheme for parabolic problems," in Numerical Solution of Partial Differential Equations II, B. Hubbard, Ed., pp. 327-350, Academic Press, New York, NY, USA, 1971. 


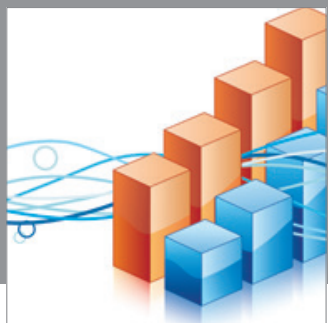

Advances in

Operations Research

mansans

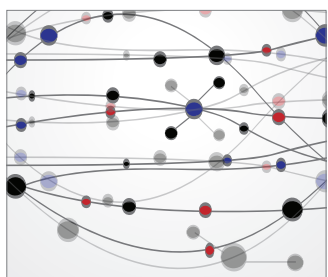

The Scientific World Journal
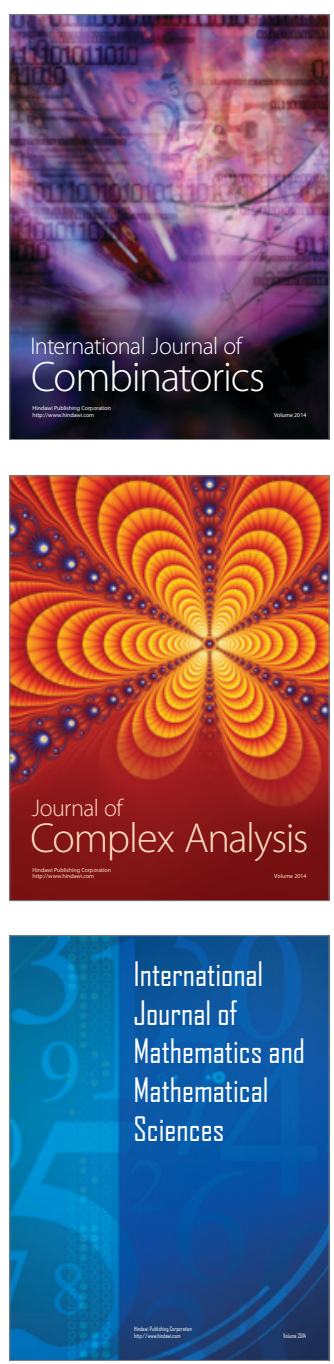
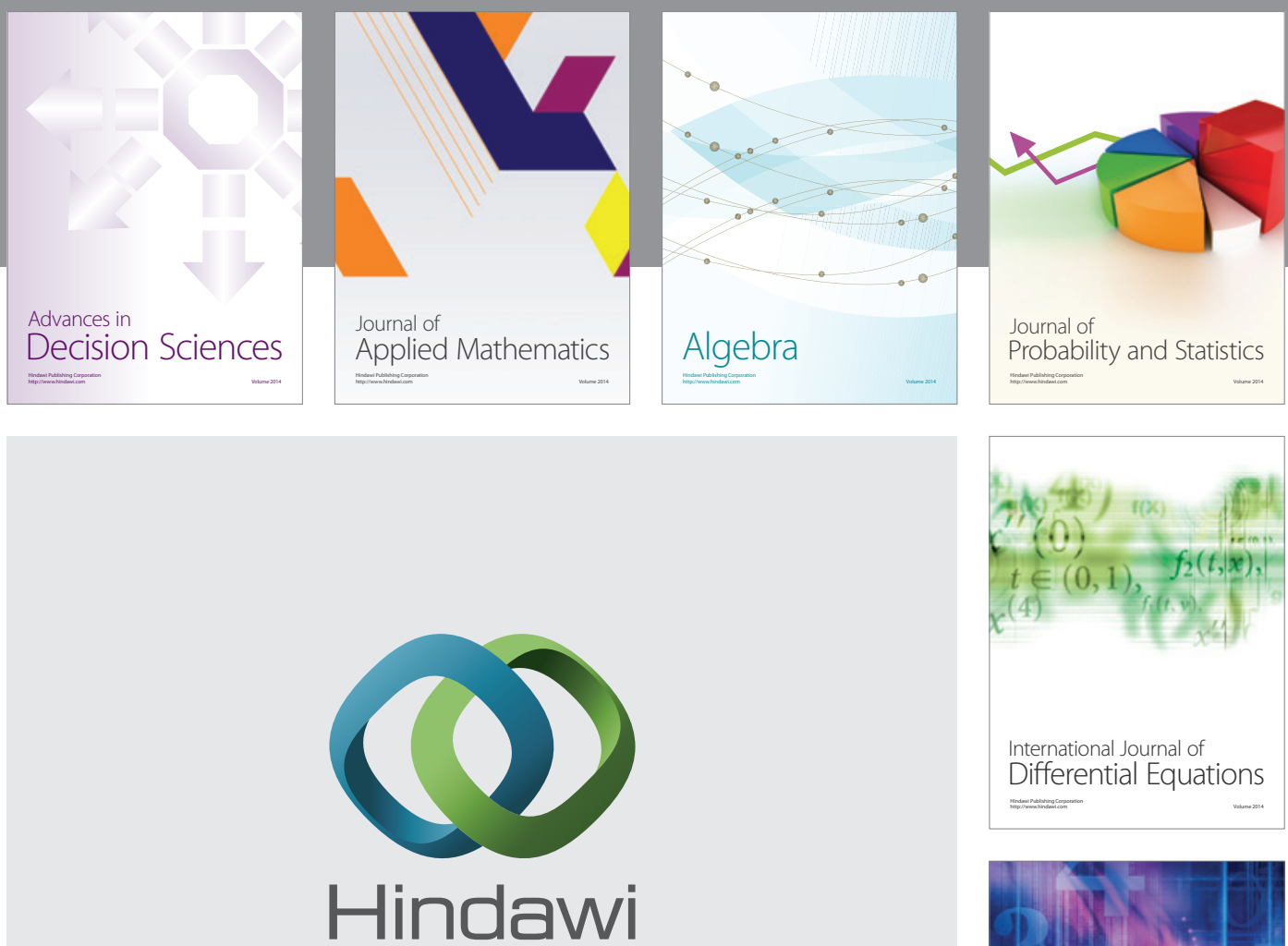

Submit your manuscripts at http://www.hindawi.com
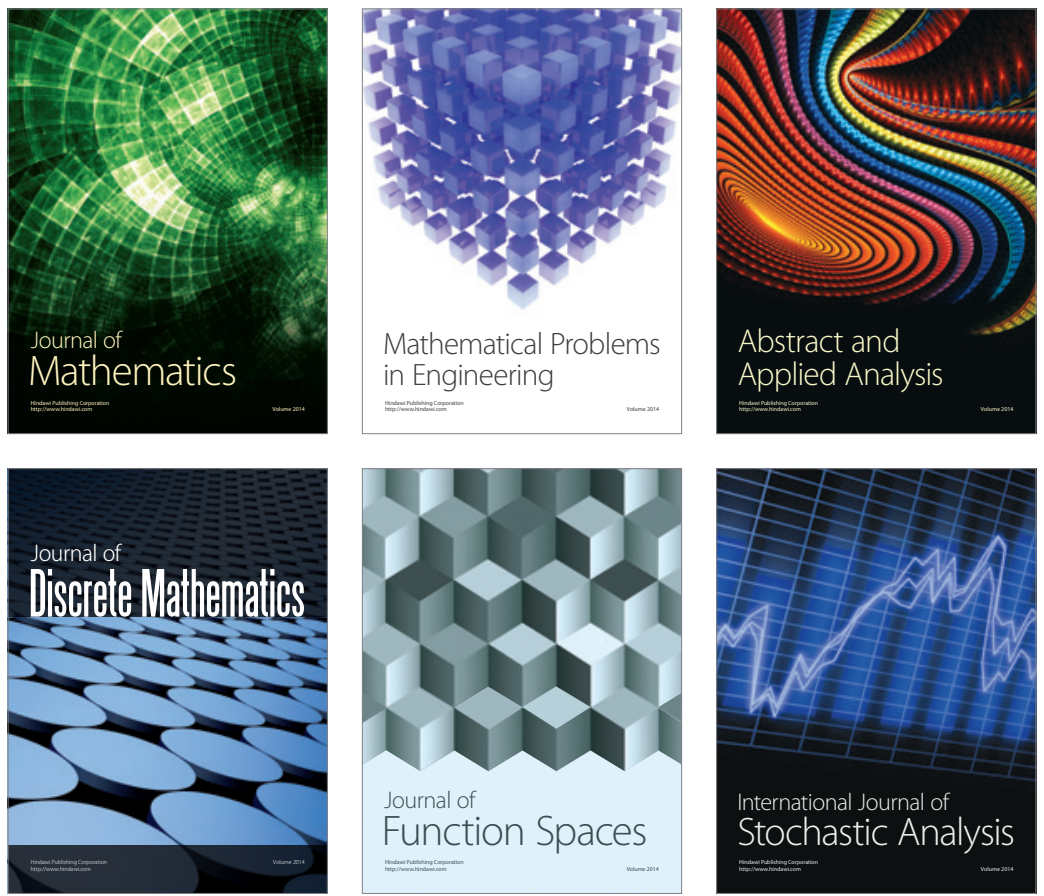

Journal of

Function Spaces

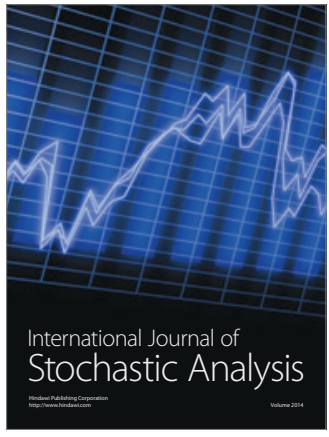

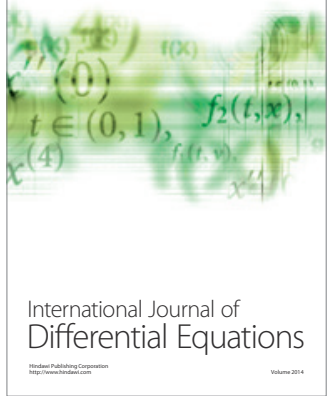
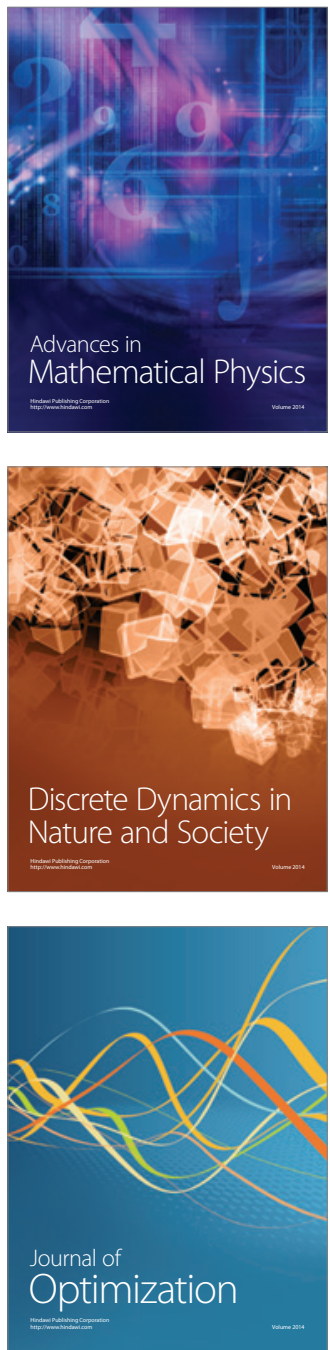